\title{
Phenotypic and Genetic Characterization of Antimicrobial Profiles of Helicobacter pylori Strains in Cuba
}

\author{
Rafael Llanes', Claudia Soria', Shigeo Nagashima², Nobumichi Kobayashi², Angela Gala', Daymi \\ Guzmán', Onelkis Feliciano', Lidunka Valdés', Oderay Gutiérrez', Heriberto Fernández³, \\ Alina Llop', and Akihiro Wada ${ }^{4}$ \\ 'Microbiology, Clinical and Epidemiology Branch, Institute Pedro Kouri, Havana, Cuba, ${ }^{2}$ Department of Hygiene, Sapporo \\ Medical University, Sapporo, Japan, ${ }^{3}$ Department of Microbiology, Universidad Austral de Chile, Valdivia, Chile, and \\ ${ }^{4}$ Department of Bacteriology, Institute of Tropical Medicine, Nagasaki University, Japan
}

\begin{abstract}
The study evaluated the antibiotic resistance patterns of Helicobacter pylori strains against metronidazole and clarithromycin in a hospital in Havana, Cuba. Eighty-five percent, 22.5\%, and 10\% of $40 \mathrm{H}$. pylori strains investigated were resistant to metronidazole, ciprofloxacin, and clarithromycin respectively but all were susceptible to amoxicillin and tetracycline. RdxA truncation was found only in metronidazole-resistant strains. In such strains, reported are eight and two novel mutations in the $r d x A$ and frxA genes respectively. Two-point mutations in the $23 \mathrm{~S} r R N A$ genes of clarithromycin-resistant strains were detected. A high prevalence of metronidazole resistance was found in Cuban $H$. pylori strains. Mutations in the $r d x A$ gene may contribute more significantly than frxA gene to the high level of resistance to metronidazole. This study supports the need to continue monitoring the antibiotic susceptibility in $H$. pylori in Cuba to guide the treatment of such infection.
\end{abstract}

Key words: Antibiotic resistance; Gene mutations; Helicobacter pylori; Cuba

\section{INTRODUCTION}

Helicobacter pylori is responsible for chronic gastritis, peptic ulcer disease, and gastric mucosa-associated lymphoid tissue lymphoma and is a major risk factor for the development of gastric adenocarcinoma (1). Eradication of such bacteria by treatment with two antimicrobial agents-clarithromycin (CLA) and amoxicillin (AMX) or metronidazole (MTZ) - and a proton pump inhibitor is recommended by various consensus groups (2).

Investigation on the susceptibility of $H$. pylori to antibiotics is one of the main factors associated with successful eradication therapy (3).

MTZ resistance in $H$. pylori is caused by null mutations in the $r d x A$ gene and less frequently by

Correspondence and reprint requests should be addressed to:

Dr. Rafael Llanes

Institute Pedro Kouri

Autopista Novia Mediodía km6

PO Box 601

Marianao 13, Havana

Cuba

Email: 1lanes@ipk.sld.cu

Fax: 5372046051 mutations in frx $A$ and $f d x B$ genes. Resistance to CLA is associated with point mutations in the 23S rRNA gene (2).

The prevalence of $H$. pylori-resistant strains is high in naive patients and even higher in patients suffering unsuccessful eradication therapy (4).

In Cuba, the prevalence of $H$. pylori-associated infection among children and adults is $42.4 \%$ and $82.7 \%$ respectively (5); however, no information is available on antimicrobial susceptibility to commonly-used drugs for the treatment of infection due to H. pylori. This study was conducted to evaluate (a) resistance of five antimicrobial agents and (b) genetic basis for MTZ and CLA resistance in $H$. pylori strains from a hospital in Havana city, Cuba. The study also investigated the demographic and clinical factors associated with antibiotic resistance.

\section{MATERIALS AND METHODS}

Patients, gastric biopsies, and culture

In total, 70 consecutive patients aged $19-68$ years were enrolled, and of them, 42 were male and 28 were female. They attended the hospital of the Institute Pedro Kouri, Havana, Cuba, for upper gastroin- 
testinal endoscopy during the last four months of 2005. Of the patients, 51 had non-ulcer dyspepsia (NUD), and 19 had peptic ulcer (PU).

A gastric biopsy specimen obtained from the antrum of each patient was cultured onto selective Columbia agar plates (Oxoid, UK), with 10\% sheepblood and Dent supplement (Oxoid). The plates were incubated at $37^{\circ} \mathrm{C}$ in a micro-aerobic atmosphere (Campybag, Oxoid) for 3-5 days. A culture was considered positive if typical $H$. pylori colonies were observed and the microorganisms grown were positive in catalase, oxidase and urease tests (5).

\section{Susceptibility testing}

The minimum inhibitory concentrations (MICs) were determined for MTZ, CLA, AMX, ciprofloxacin (CIP), and tetracycline (TET) by the E-test method (AB Biodisk, Solna, Sweden) as described by the Clinical and Laboratory Standards Institute (CLSI). Mueller Hinton agar (Oxoid), with 5\% of aged sheep-blood, was used as culture medium for determining antibiotic susceptibility (6). All tests were performed thrice, and H. pylori ATCC 43504 was used as a control strain.

Susceptibility to CLA was interpreted according to the guidelines of the CLSI (6). Since the CLSI has not designated breakpoints for other antimicrobials in $H$. pylori, the following MIC values were used in defining resistance: $\mathrm{MTZ} \geq 8 \mathrm{mg} / \mathrm{L}, \mathrm{AMX}$ and CIP $\geq 1 \mathrm{mg} / \mathrm{L}$, and TET $\geq 2 \mathrm{mg} / \mathrm{L}$ (7).

\section{PCR and DNA sequence analysis}

Bacterial genomic DNA was extracted using the DNeasy tissue kit (QIAGEN, Japan). Resistant genes - $r d x A$, fr $x A$, and 23S $r R N A$-were amplified using PCR with specific primers as previously described $(8,9)$. Amplified DNA was purified using Wizard-SV gel and PCR Clean-Up System (Promega, USA) and was then sequenced in both directions using the ABI PRISM 3100 automatic sequencer (Applied Biosystems, USA). For identification of ribosomal mutations, sequences were compared with the genomic sequences of $H$. pylori reference strains J99 and 26695 (8). Comparisons were done using the software available over the Internet at the National Center for Biotechnology Information website (http://www.ncbi.nlm.nih.gov), and multiple alignments were performed using the GenetyxMac software (version 11.2) (Genetyx Corporation, Japan) (10).

\section{Statistical analysis}

Statistical analysis was carried out with the SPSS software for Windows (version 11.0) (Chicago, USA). The chi-square and Fischer's tests were used for determining the differences between patterns of resistance by age-group, sex, and clinical features. The $p$ values of $<0.05$ were regarded as statistically significant.

\section{Ethics}

The present study was conducted in accordance with the Declaration of Helsinki and the ethical committee of the Institute Pedro Kouri. Written informed consent was obtained from each subject to participate in the study. The gastric mucosa biopsy for diagnosis of $H$. pylori-associated infection is part of the routine management in patients with dyspepsia and peptic ulcer.

\section{RESULTS}

\section{Antimicrobial susceptibility}

Forty-six (65.7\%) of the 70 enrolled patients were positive for $H$. pylori by culture. Of the 46 strains, 40 were available for antimicrobial susceptibility testing with $67.5 \%$ (27 of 40 ) and $32.5 \%$ (13 of 40 ) of the strains from non-ulcer dyspepsia and peptic ulcer patients respectively.

Eighty-five percent, $22.5 \%$, and $10 \%$ of the $40 \mathrm{H}$. pylori strains investigated were resistant to MTZ, CIP, and CLA respectively but all were susceptible to AMX and TET. The $\mathrm{MIC}_{50}$ and $\mathrm{MIC}_{90}$ values for MTZ were $256 \mathrm{mg} / \mathrm{L}$. In the case of CIP, the $\mathrm{MIC}_{50}$ was $0.125 \mathrm{mg} / \mathrm{L}$, and the $\mathrm{MIC}_{90}$ was $32 \mathrm{mg} / \mathrm{L}$ (Table $1)$.

Our study revealed multiple antibiotic resistance patterns in $25 \%(10 / 40)$ of $H$. pylori strains investigated. Combined resistance to MTZ-CIP (6/10 strains) was most frequently found, followed by patterns-MTZ-CLA and MTZ-CIP-CLA, with two strains each.

Antimicrobial susceptibilities of the strains collected from patients with PU and NUD, males and females and stratified in age-groups below and above 40 years were compared, and no significant difference $(\mathrm{p}>0.05)$ in antimicrobial resistance was observed among these groups (Table 2).

\section{Characterization of $r d x A$ and frxA genes of MTZ-sensitive and resistant strains}

A MTZ-sensitive strain (\#56) exhibited amino acid substitution (missense mutation). In the case of MTZ-resistant strains, one had a missense mutation (\#191); seven demonstrated amino acid substitutions (\#84C, \#93, \#113, \#114, \#127, \#227, and \#230); two had nonsense mutations (\#81 and \#117); and three others (\#67, \#84A, and \#146) showed both nonsense mutations and amino acid 


\begin{tabular}{|c|c|c|c|c|c|c|c|}
\hline \multirow{3}{*}{ Antimicrobial } & \multicolumn{4}{|c|}{ Antimicrobial susceptibility } & \multirow{2}{*}{\multicolumn{3}{|c|}{ MIC values (mg/L) }} \\
\hline & \multicolumn{2}{|c|}{ Susceptible } & \multicolumn{2}{|c|}{ Resistant } & & & \\
\hline & No. & $\%$ & No & $\%$ & MIC range & $\mathrm{MIC}_{50}$ & $\mathrm{MIC}_{90}$ \\
\hline Amoxicillin & 40 & 100 & - & & $0.016-1$ & 0.016 & 0.03 \\
\hline Clarithromycin & 36 & 90 & 4 & 10 & $0.016-256$ & 0.016 & 0.125 \\
\hline Ciprofloxacin & 31 & 77.5 & 9 & 22.5 & $0.016-32$ & 0.125 & 32 \\
\hline Metronidazole & 6 & 15 & 34 & 85 & $1-256$ & 256 & 256 \\
\hline Tetracycline & 40 & 100 & - & & $0.016-1$ & 0.06 & 0.25 \\
\hline
\end{tabular}

Table 2. Resistance of 40 Helicobacter pylori Cuban strains to three antibiotics by sex, age, and clinical features of patients

\begin{tabular}{|c|c|c|c|c|c|c|}
\hline \multirow[b]{2}{*}{ Antimicrobial } & \multicolumn{6}{|c|}{ No. (\%) of resistant strains in patients } \\
\hline & $\frac{P U}{(n=13)}$ & $\begin{array}{c}\text { NUD } \\
(\mathrm{n}=27)\end{array}$ & $\begin{array}{c}\text { Male } \\
(n=22)\end{array}$ & $\begin{array}{c}\text { Female } \\
(\mathrm{n}=18)\end{array}$ & $\begin{array}{c}<40 \text { years } \\
(\mathrm{n}=13)\end{array}$ & $\begin{array}{c}>40 \text { years } \\
(\mathrm{n}=27)\end{array}$ \\
\hline \multirow[t]{2}{*}{ Metronidazole } & $11(84.6)$ & $23(85.2)$ & $21(95.4)$ & $13(72.2)$ & $11(84.6)$ & $23(85.2)$ \\
\hline & \multicolumn{2}{|c|}{$\mathrm{p}=0.67$} & \multicolumn{2}{|c|}{$\mathrm{p}=0.10$} & \multicolumn{2}{|c|}{$\mathrm{p}=0.67$} \\
\hline \multirow[t]{2}{*}{ Clarithromycin } & $1(7.7)$ & $3(11.1)$ & $3(13.6)$ & $1(5.6)$ & - & $4(14.8)$ \\
\hline & \multicolumn{2}{|c|}{$\mathrm{p}=0.82$} & \multicolumn{2}{|c|}{$\mathrm{p}=0.75$} & \multicolumn{2}{|c|}{$\mathrm{p}=0.36$} \\
\hline \multirow[t]{2}{*}{ Ciprofloxacin } & $2(15.4)$ & $7(25.9)$ & $5(22.7)$ & $4(22.2)$ & $2(15.4)$ & 7 (25.9) \\
\hline & \multicolumn{2}{|c|}{$\mathrm{p}=0.73$} & \multicolumn{2}{|c|}{$p=0.73$} & \multicolumn{2}{|c|}{$p=0.73$} \\
\hline
\end{tabular}

substitutions that resulted in a truncated RdxA protein at positions $35,75,139$, and 211 . One strain (\#17) remained unchanged for the $r d x A$ gene (Table $3)$.

With a few exceptions, the open reading frame for the frxA gene was disrupted by a deletion of a nucleotide (nt) at position 54 and 98 , and in the resistant strain \#146 by a nt insertion at position 248. These events led to the occurrence of a stop codon at positions corresponding to amino acids 39,74 , or 83 . In resistant strains \#113 and \#114, some point mutations were observed (Table 3 ).

\section{Analysis of mutations in CLA-resistant and susceptible strains}

Of the three resistant strains investigated, two (66.7\%) harboured 23S rDNA mutations at position A2142G $(n=1)$, or in both, A2142 and A2143 positions $(n=1)$. These two strains exhibited high-level resistance to CLA (MIC $>256 \mathrm{mg} / \mathrm{L}$ ). The remaining three strains (two susceptible and a CLA-resistant one with an MIC value of $8 \mathrm{mg} / \mathrm{L}$ ) did not show such alterations at the conserved $\mathrm{V}$ domain of genesencoding $23 S$ rRNA (data not shown).

\section{DISCUSSION}

In patients undergoing endoscopy, therapy should be tailored based on antimicrobial susceptibility data (11).
Resistance to antimicrobials, as detected in culture, is of particular concern with $H$. pylori as a major cause of eradication failure. Resistance to MTZ is the most common type of resistance in this pathogen (3). The high frequency of resistance to this drug in $H$. pylori strains studied might be due to its frequent use for the treatment of intestinal parasites and gynaecological disorders. MTZ also represents the third most-used antibiotic by Cuban patients attending the primary healthcare system (12).

It is noteworthy that the mutation Ala (37) $\rightarrow$ Val, detected in a MTZ-susceptible strain, was different from those observed in MTZ-resistant ones, and so could not be essential for resistance. Some mutations detected in the $r d x A$ gene from the resistant strains-Arg(10) $\rightarrow$ Ile; $\operatorname{Arg}(16) \rightarrow$ Cys; $\operatorname{Arg}(16) \rightarrow$ His; His $(97) \rightarrow$ Thr; Ser(81) $\rightarrow$ Leu-have been described previously $(8,9,13)$.

An interesting finding in MTZ-resistant strains in our study was the detection of several mutations not previously described in the literature in $r d x A$ gene: [Glu (139) $\rightarrow$ Stop codon; Pro(106) $\rightarrow$ Ser; $\mathrm{Val}(111) \rightarrow$ Ala; $\operatorname{Thr}(208) \rightarrow$ Ala; Asn(14) $\rightarrow$ Thr; Glu $(171) \rightarrow$ Lys; $\operatorname{Met}(56) \rightarrow$ Val; $\operatorname{Arg}(191) \rightarrow$ Lys] and in frxA gene: [Glu(199) $\rightarrow$ Stop codon; Stop codon at position 83], which might be associated with resistance. 


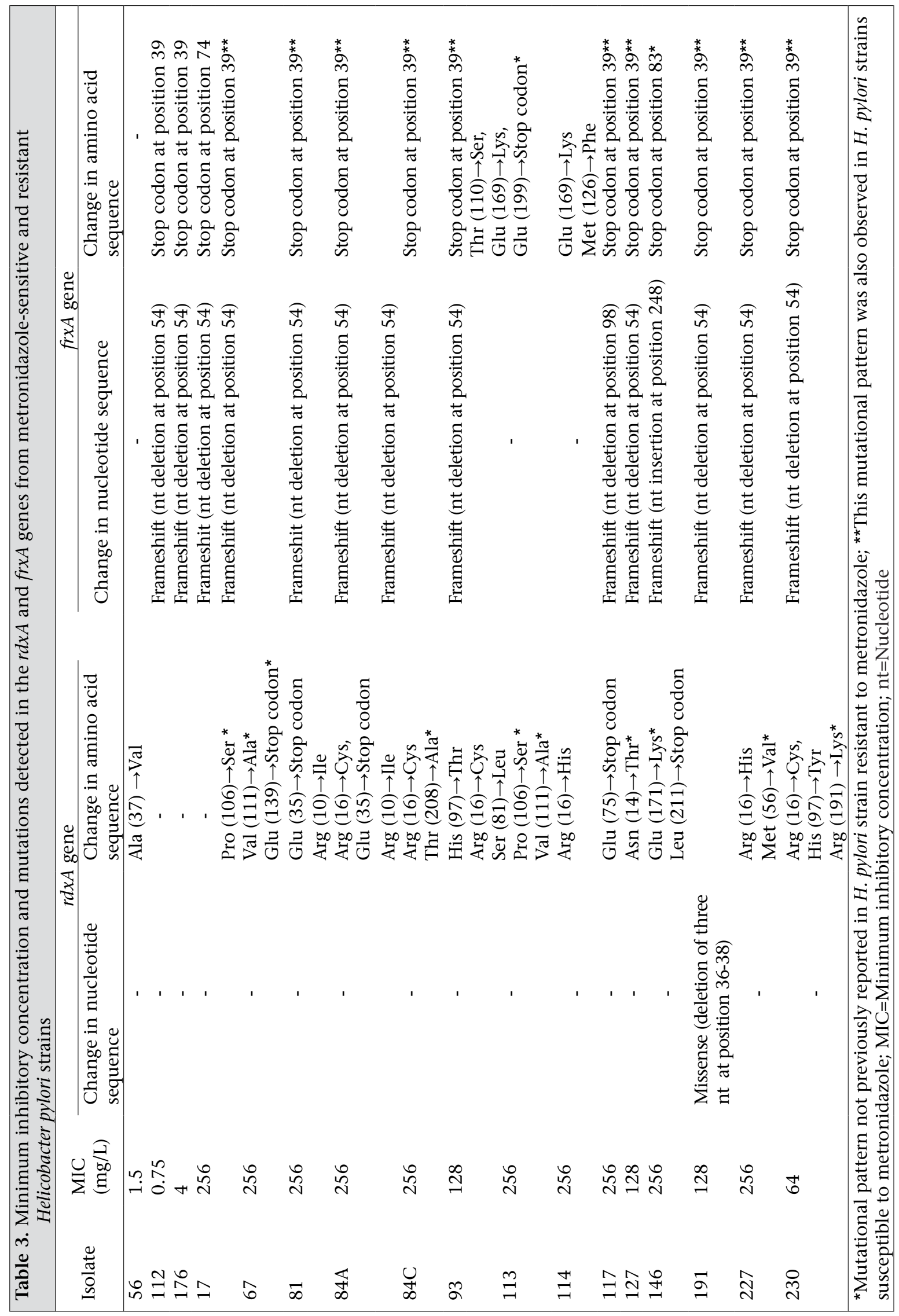


Some reports established that inactivation of fr $x A$ without mutations in $r d x A$ could not cause MTZ resistance $(8,13)$. Our results suggest that alterations of frxA alone produced resistance, e.g. strain 17. However, as the frxA mutations were also observed in MTZ-susceptible strains, we conclude that these are unlikely to contribute to the MTZ resistance of these strains.

CLA is the most powerful antibiotic currently used for the treatment of $H$. pylori. Resistance to this antibiotic considerably reduces the success rate of standard triple therapies (14). The percentage of CLA resistance found in this study and the type of mutations in the 23S rRNA gene are similar to reports from the USA, Japan, and Bulgaria (2).

In the current investigation, a moderately-resistant CLA strain lacked any mutation in the analyzed region of the 23S rRNA gene, and the basis for such resistance remains undetermined. It suggests that other undetected $23 S$ rRNA mutations may be involved in resistance, which has been reported elsewhere (15). As $H$. pylori contains two copies of $23 S$ $r R N A$, sometimes it exhibits a heterozygous condition in which one of the genes is mutated and the other remains normal. This genotype confers a CLA-resistant phenotype (15). In addition, a heterogeneous resistance pattern reflecting mixed infections with susceptible and resistant populations to CLA and other antimicrobials has been observed in $H$. pylori isolates (16).

CLA has never been used for the treatment of $H$. pylori-associated infections in Cuba but other macrolides, such as erythromycin and azithromycin, have been widely used in several infections in this country (17). Cross-resistance between CLA and other macrolides have been described before and may account for the observed resistance (14).

Combined resistance to MTZ and CLA may compromise the effectiveness of current triple therapy regimens for $H$. pylori-associated infections (16). Interestingly, our analysis of resistance patterns showed that strains with dual resistance to these drugs were relatively uncommon (10\%) in the study population.

After treatment failures to commonly-used anti- $H$. pylori antibiotics, such as MTZ or CLA, quinolonebased triple therapies have been proposed as rescue regimens (2). The prevalence of resistance to such antimicrobials has been determined in only a limited number of studies $(7,18)$. The mechanism of resistance to fluoroquinolones in $H$. pylori has been shown to be linked to mutations in the so-called quinolone resistance-determining region (QRDR) of the gyrA gene (18). In the present study, we did not investigate the mutations associated with such resistance. In future studies, it would be noticeable to perform the sequencing analysis of the QRDR region in fluoroquinolone-resistant strains. The high percentage of CIP resistance in the present study might be a consequence of its frequent use for the management of several infectious diseases in Cuba (12).

In vitro resistance to AMX or TET appears to be rare among primary isolates of $H$. pylori (13) as, in our study, several investigations have shown a very marked susceptibility to these two drugs $(2,5,14)$. However, it is necessary to mention that subsequent freezing of $H$. pylori strains at $-80{ }^{\circ} \mathrm{C}$ can result in the loss of AMX-resistant phenotype (19).

The present current study was conducted to gather preliminary data on antimicrobial susceptibility of H. pylori strains from a hospital in Havana, Cuba. Consequently, they are not representative of all $\mathrm{Cu}$ ban population. The participating hospital centre serves only adult patients from a limited area.

In resume, we found a high frequency of MTZ and CIP resistance in $H$. pylori strains for the first time in Cuba. Mutations in the $r d x A$ gene may contribute more significantly than frxA gene to the high-level MTZ resistance. The present investigation suggests the need for continuous monitoring of the antimicrobial susceptibility in $H$. pylori strains in Cuba.

\section{Public-health implications}

Overall, the weight of evidence suggests that eradication of $H$. pylori will prevent the majority of gastric cancers that are caused by gastric inflammation and many that are associated with gastric atrophy. Screening of population and treatment for infection due to $H$. pylori is an appealing strategy (20) as the aim is to prevent disease and its complications. The data on antimicrobial susceptibilities provided by the present study is critical to guide the clinicians on the effectiveness of treatment regimens.

\section{ACKNOWLEDGEMENTS}

The study is supported, in part, by the Canadian International Development Agency (CIDA) through the Global Health Research Initiative (File: 102172006).

\section{REFERENCES}

1. Zhang YY, Xia HH, Zhuang ZH, Zhong J. True re-infection of Helicobacter pylori after successful eradication-worldwide annual rates, risk factors and clinical 
implications. Aliment Pharmacol Ther 2008;29:14560.

2. Mégraud F. Helicobacter pylori antibiotic resistance: prevalence, importance, and advances in testing. Gut 2004;53:1374-84.

3. Nahar S, Mukhopadhyay AK, Khan R, Ahmad MM, Datta S, Chattopadhyay S et al. Antimicrobial susceptibility of Helicobacter pylori strains isolated in Bangladesh. J Clin Microbiol 2004;42:4856-9.

4. Romano M, Iovene MR, Russo MI, Rocco A, Salerno $\mathrm{R}$, Cozzolino D et al. Failure of first-line eradication treatment significantly increases prevalence of antimicrobial-resistant Helicobacter pylori clinical isolates J Clin Pathol 2008;61:1112-5.

5. González-Carbajal M. Helicobacter pylori el tercer dogma. 1st ed. Barcelona: Doyma, 2004:120-59.

6. Clinical and Laboratory Standards Institute. Performance standards for antimicrobial susceptibility testing; fifteenth informational supplement. Wayne: Clinical and Laboratory Standards Institute, 2007:134. (M100-S17).

7. Bogaerts P, Berhin C, Nizet H, Glupczynski Y. Prevalence and mechanisms of resistance to fluoroquinolones in Helicobacter pylori strains from patients living in Belgium. Helicobacter 2006;11:441-5.

8. Yang YJ, Wu JJ, Sheu BS, Kao AW, Huang AH. The $r d x A$ gene plays a more major role than frxA gene mutation in high-level metronidazole resistance of Helicobacter pylori in Taiwan. Helicobacter 2004;9:4007.

9. Wang G, Wilson TJM, Jiang Q, Taylor DE. Spontaneous mutations that confer antibiotic resistance in Helicobacter pylori. Antimicrob Agents Chemother 2001;45:727-33.

10. Ogawa Y, Fukutomi T, Sugiura K, Kato K, Tohya Y, Akashiet H. Comparison of Akabame virus isolated from sentinel cattle in Japan. Vet Microbiol 2007;124:16-24.

11. Romano M, Marmo A, Cuomo A, De Simone T, Mucherino C, Iovene et al. Pretreatment antimicrobial susceptibility testing is cost saving in the eradica- tion of Helicobacter pylori. Clin Gastroenterol Hepatol 2003;1:273-8.

12. Lara MC, Cires M, Garcia AJ. Consumo de antimicrobianos en Atención primaria de salud. Rev $\mathrm{Cu}$ bana Med Gen Integr 2003;19:2-6.

13. Kwon DH, Hulten K, Kato M, Kim JJ, Lee M, ElZaatari FAK et al. DNA sequence analysis of $r d x A$ and frxA from 12 pairs of metronidazole-sensitive and resistant clinical Helicobacter pylori isolates. Antimicrob Agents Chemother 2001;45:2609-15.

14. Duck WM, Sobel J, Pruckler JM, Song Q, Swerdlow $\mathrm{D}$, Friedman $\mathrm{C}$ et al. Antimicrobial resistance, incidence and risk factors among Helicobacter pyloriinfected persons, United States. Emerg Infect Dis 2004;10:1088-94.

15. Posteraro P, Branca G, Sanguinetti M, Ranno S, Cammarota G, Rahimi S et al. Rapid detection of clarithromycin resistance in Helicobacter pylori using a PCR-based denaturing HPLC assay. JAC 2006; 57:718.

16. De Francesco V, Margiotta M, Zullo A, Hassan C, Valle ND, Burattini $\mathrm{O}$ et al. Primary clarithromycin resistance in Italy assessed on Helicobacter pylori DNA sequences by TaqMan real-time polymerase chain reaction. Aliment Pharmacol Ther 2006;23:429-35.

17. Llanes R, Sosa J, Guzmán D, Llop A, Valdés EA, Martínez I et al. Antimicrobial susceptibility of Neisseria gonorrhoeae in Cuba (1995-1999). Implications for treatment of gonorrhea. Sex Transm Dis 2003;30:10-4.

18. Cattoir V, Nectoux J, Lascols C, Deforges L, Delchier JC, Megraud F et al. Update on fluoroquinolone resistance in Helicobacter pylori: new mutations leading to resistance and first description of a gyrA polymorphism associated with hypersusceptibility. Int J Antimicrob Agents 2007;29:389-96.

19. Dore MP, Osato MS, Realdi G, Mura I, Graham DY, Sepulveda AR. Amoxycillin tolerance in Helicobacter pylori. JAC 1999;43:47-54.

20. Moayyedi P, Hunt RH. Helicobacter pylori. Public health implications. Helicobacter 2004;9:67-72. 